Saudi Journal of Biomedical Research

Abbreviated Key Title: Saudi J Biomed Res ISSN 2518-3214 (Print) |ISSN 2518-3222 (Online)

\title{
Prevalence of Intestinal Parasitic Infections among Primary Schools aged Children in Ombda Locality
} \author{
Ahmed $^{3}$ \\ ${ }^{1}$ Medical Parasitology department, FMLS, Alneelain University Sudan \\ ${ }^{2}$ Head Department, Medical Parasitology Department, Faculty of Health Science, Elsheikh Abdallah Elbadri University \\ ${ }^{3}$ Professor of Medical Parasitology department, FMLS, Omdurman Alhlia University Bahrain
}

Abuobieda Sirelkhatem Mohammed Elameen ${ }^{1 *}$, Mosab Nouraldein Mohammed Hamad ${ }^{2}$, Mohammed Baha Eldin

DOI: $10.36348 /$ SJBR.2019.v04i12.001

| Received: 02.12.2019 | Accepted: 10.12.2019 | Published: 14.12.2019

*Corresponding author: Abuobieda Sirelkhatem Mohammed Elameen

Abstract

210 stool samples were collected from primary school children (100 male and 110 female).The stool samples were processed by wet prepration, formal ether concentration technique and saturated sugar flotation technique. The result revealed that out of 210 stool samples, 52 were found positive for intestinal parasites. This constituted an overall prevalence rate of $24.8 \%$. The study revealed that the prevalence of intestinal parasites was $29 \%$ among males while it was $20.9 \%$ among females. The highest prevalence rate $(35 \%)$ was reported among the 6-8 years age groups, while the lowest prevalence rate $(10.3 \%)$ was reported among $12-14$ years age groups. The prevalence rate of different parasites was as follow: Giardia lamblia (16.6\%), Hymenolepis nana (6.7\%), Taenia species (0.5\%), Ascaris lumbericoides $(0.5 \%)$ and Entamoeba histolytica $(0.5 \%)$. The prevalence of intestinal protozoa $(17.1 \%)$ was higher than the intestinal helminthes $(7.6 \%)$. The study showed that $23.3 \%$ were infected with single infection and $1.4 \%$ was infected with mixed infection. Formal ether concentration technique was considered as the gold standard in detection of intestinal parasites. The sensitivity and specificity of saturated sugar flotation technique were $75 \%$ and $100 \%$ respectively

Keywords: intestinal parasites, infection, school children.

Copyright @ 2019: This is an open-access article distributed under the terms of the Creative Commons Attribution license which permits unrestricted use, distribution, and reproduction in any medium for non-commercial use (NonCommercial, or CC-BY-NC) provided the original author and source are credited.

\section{INTRODUCTION}

Intestinal parasitic infections (IPIs) are among the most prevalent of human parasitic infections worldwide, causing significant morbidity and mortality. More than 3.5 billion people are affected, and that 450 million are ill as a result of these infections, the majorities are being children. Up to 250 million people are estimated to be infected with at least one or more species of intestinal nematodes in Sub-Saharan African countries. School age children and pregnant women are among the high risk groups for intestinal parasitic infections. These infections are also known as serious public health problems because the complications such as iron deficiency anemia, growth retardation in children and other physical and mental health problems with serious consequences may occur [1].

Intestinal parasitic infections are the main health problems which can cause mortality and morbidity among infected people. They are also associated with stunting of linear growth, physical weakness and low educational achievement in children.
Moreover, they cause iron deficiency anemia, loss of appetite and other physical and mental problems [2].

Gastrointestinal parasites (GIPs) and severity of its infections have a profound impact on human public health and development, affecting approximately one-third of the world's population, causing high mortality rate mostly in children [3].

Intestinal parasitosis, a major public health problem in developing countries is aggravated by hot and humid climate, poverty, malnutrition, high population density, and poor health. Multiple socioeconomical, cultural, physiological and behavioral parameters along with illiteracy and poor sanitation influence intestinal parasitic infection [4].

Despite considerable effort to prevent and control intestinal parasitic infections but still remain endemic in many West African countries and Nigeria inclusive. the disease has a lot of significance and public health consequences with school age children according to an estimate, million are ill while billions 
are already infected as a result of these neglected tropical diseases (NTDs) which mostly are school children in which the burden of morbidity and mortality are high in developing countries. Intestinal parasites and soil transmitted helminthes are common and affects the vulnerable group in the community such as children and pregnant women and the diseases are mostly found among the rural areas of the developing countries of Africa, Asia and Central America which are often associated with lack of safe water supply and poor sanitation mostly affected [5].

Clinical manifestation among children harboring these parasites include abdominal pain, nausea, reduced appetite, iron-deficiency anaemia, retarded growth and impaired cognitive performance [6]. Intestinal parasitic infestations are the most common consequences have been shown to cause nutritional status, physical development mental functions, and verbal ability and inhibition control aspects of cognitive behavior of the children [6]. Parasitic worms are the commonest infections particularly in children and adults, which estimates more than 3 billion worm infections in the world [7].

\section{Rationale}

Parasitic intestinal infection is an important cause of morbidity and mortality in developing world, no firm and consistent policy exists on the control of intestinal infections compared to policies already in place on diseases such as malaria and filariasis. Intestinal parasites have been shown to produce a detrimental effect on cognition and educational achievement in children.

\section{OBJECTIVES}

\section{General objectives}

To determine prevalence of intestinal parasites among the school children in Ombda locality, Khartoum state

\section{Specific objectives}

- To determine prevalence of intestinal parasites among the school children in the study area according to age and gender.

- To identify the types of parasites prevalent among the school children in the study areas.

- To compare between formal ether concentration technique and saturated sugar flotation technique.

- To identify the major intestinal parasites in the study area.

\section{MATERIALS AND METHOD}

Study design

Descriptive cross sectional study.

\section{Study area}

The study was performed in five primary schools in Ombada locality which is considered part of Omdurman city, Khartoum state.

\section{Study population}

The study was conducted on male and female primary schools children in Ombada locality.

\section{Sample size}

210 subjects were included in this study.

\section{Ethical consideration}

Informed consents were taken from all of the participants or their gardeners after explaining the nature of the study.

\section{STATISTICAL ANALYSIS}

Data were analyzed using Statistical Package for the Social Scieces (SPSS) software. Statistical analysis was done using Chi-square to evaluate any association between parasitic infections.

\section{Samples reception and processing}

Sterile plastic containers were distributed which were labeled with the name and an assigned code to collect the samples; information and explanations were furnished to how they were to collect and transport the stool samples to the collection sites. The collection and transportation guidelines followed preanalytical standard operating procedures (SOPs).

\section{METHODOLOGY \\ Wet preparation}

One drop of $0.85 \% \mathrm{NaCl}$ was placed on the left side of the slide and 1 drop of iodine, (working solution) was placed on the right side of the slide. Avery small amount of fecal specimen was taken (about the amount picked up on the end of an applicator stick when introduced into the specimen), and thoroughly the stool was emulsified in the saline and iodine preparations (use separate sticks for each). A cover slip ( 22 by $22 \mathrm{~mm}$ ) was placed on each suspension. Both suspensions were systematically scanned with the 10 objective. The entire cover slip area was examined. The 40 objective was used for more-detailed study. At least, one-third of the cover slip was examined with the 40 objective, even if nothing suspicious has been seen. 


\section{Formal-ether concentration technique}

One gram of stool specimen was added to $8 \mathrm{ml}$ of $10 \%$ formalin in a small beaker and thoroughly emulsified, and brought into suspension. Next the suspension was strained through a double layer of wet gauze directly into a $15 \mathrm{ml}$ centrifuge tube and the gauze was then discarded. Then $3 \mathrm{ml}$ of diethyl ether was added to the suspension in the tube, rubber stopper and shaken vigorously for 10 seconds. The content was centrifuged by using swinging bucket at 2000rpm for 3 minutes; the supernatant (comprising the top 3 layers) was decanted; and then the deposit/sediment was resuspended with a disposable Pasteur pipette. A few drops of the suspension was transferred onto a microscope slide and covered with a cover slip. Finally the preparation was examined using the low power (X10) objective, and in a systematic manner as to observe the entire cover slip area. If an organism or suspicious objects are seen, the higher magnification ( $X$ 40objective) was used to observe its detailed morphology.

\section{Saturated sugar flotation technique}

Sheather, s sugar solution was placed in cup. One gram of stool was added to solution. The fecal sample was mixed with the flotation solution. The fecal debris was screened to another cup. The filtered preparation was poured into a tube. Fecal flotation solution was added to the top of the tube .Cover slip was placed on the top of the tube. The tube was left undisturbed for 15 minutes and carefully the cover glass was placed on slide and examined microscopically.

\section{RESULT}

The results showed that out of 210 faecal samples examined, 52 were found positive for gastrointestinal parasites. This constituted an overall prevalence rate of $24.8 \%$. The study revealed that the prevalence of intestinal parasites was increasing in male $(29 \%)$ than female $(20.9 \%)$.

The prevalence of different parasites was found as follows: Giardia lambelia (16.6\%), Hymenolepis nana $(6.7 \%)$, Taenia spp $(0.5 \%)$, Ascaris lumbercoides (0.5\%), and Entamoeba histolytica $(0.5 \%)$.

The prevalence of intestinal parasites by different parasitological techniques was as follow: $21.9 \%$ by direct preparation, $24.8 \%$ by formal ether concentration technique and $18.6 \%$ by saturated sugar flotation technique.

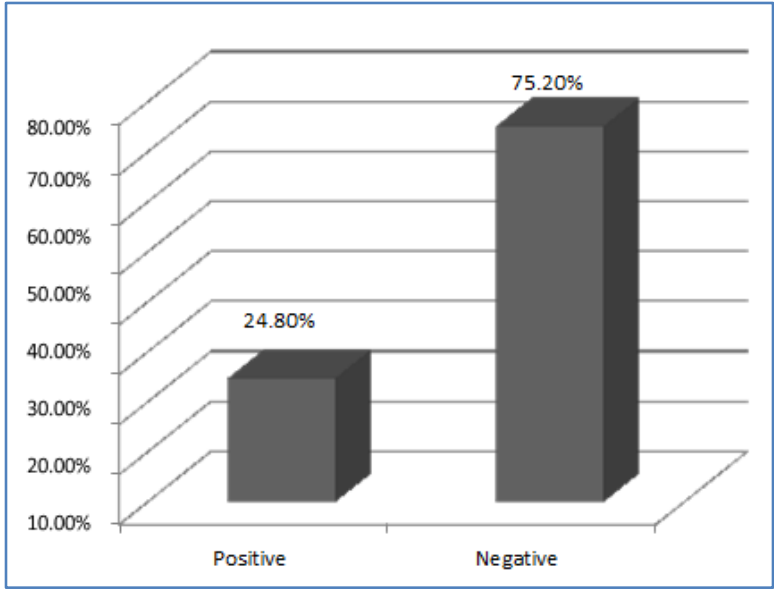

Graph-1: Showed the prevalence rate of intestinal parasites among primary schools aged children in Ombada locality

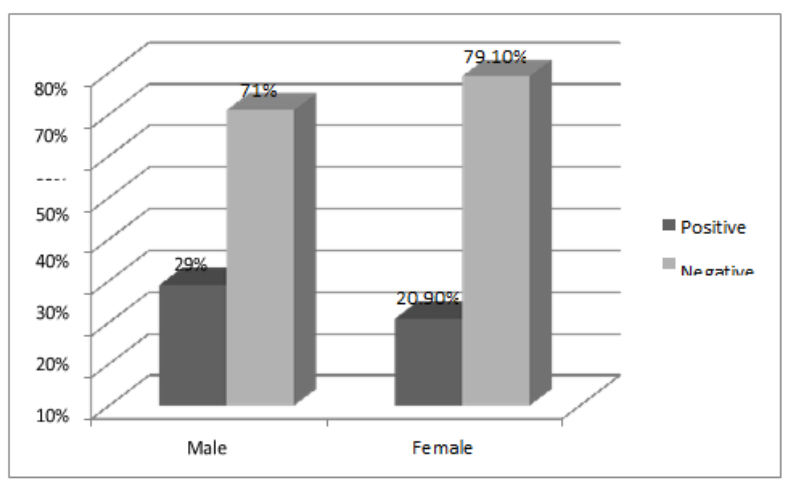

Graph-2: Showed the prevalence rate of intestinal parasites among primary schools children in Ombada locality according to gender

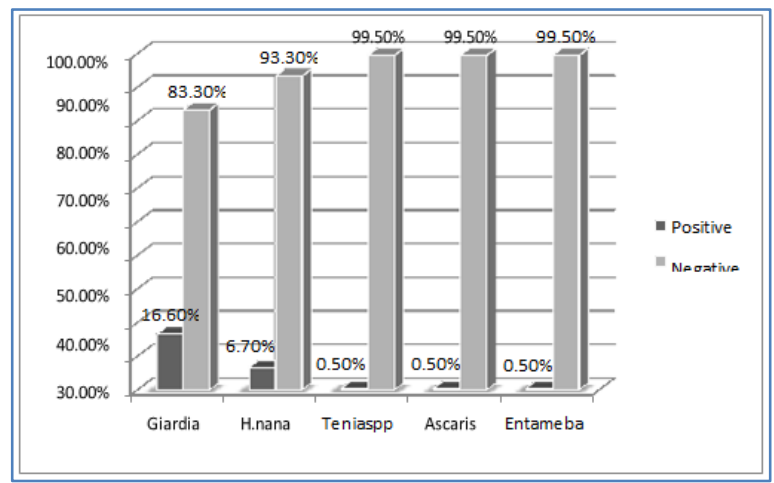

Graph-3: Showed distribution of different intestinal parasites detected among primary schools children in Ombada locality

\section{CONCLUSION AND RECOMMENDATIONS}

We conclude that formol ether centrifugation concentration techniques are the best method to detect intestinal infections, which is more common in male group. Further studies should be done parasitic with large sample size and more diagnostic methods.

\section{REFERENCES}

1. Tulu, B., Taye, S., \& Amsalu, E. (2014). Prevalence and its associated risk factors of intestinal parasitic infections among Yadot primary school children of South Eastern Ethiopia: a crosssectional study. BMC research notes, 7(1), 848. 
2. Mary, M.W., \& Anne, A. (2001). Thesis of Master of Science in human nutrition foods and exercise, 18.

3. Childers, K. A. G. (2014). A survey of the prevalence of gastrointestinal parasites and associated risk factors in children in a rural city of the Dominican Republic (Doctoral dissertation, Virginia Tech).

4. Ubachukwu, P. O. Prevalence of Intestinal Parasites among School Children in Uzo-Uwani Local Governemnt Area of Enugu State.

5. Mohammed, K., Abdullah, M. R., Omar, J., Eugene, I. I., \& Ismail, A. (2015). Intestinal parasitic infection and assessment of risk factors in North-Western, Nigeria: A community based study. International Journal of Pharma Medicine and Biological Sciences, 4(2), 141.

6. Banke, R. O., Omudu, E. A., Ikenwa, D. A., \& Feese, E. J. (2006). Prevalence of gastro-intestinal parasites in relation to availability of sanitary facilities among schooling children in makurdi, Nigeria. Animal Research International, 3(2), 489493.

7. Rayapu, V., Dhandapany, S. P., Shaker, I. A., \& Kasukurthy, S. (2012). Prevalence of Intestinal Helminthic Parasites in School Going Children in Rural Area of Kuppam, Andhra Pradesh. International Journal of Basic Medical Sciences and Pharmacy (IJBMSP), 2(2). 